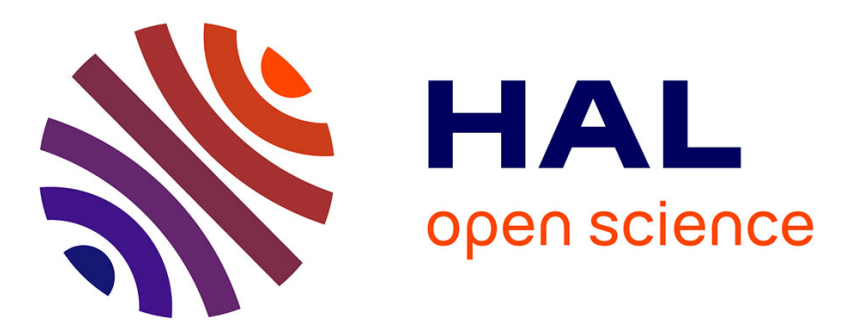

\title{
Quasi unidimensional growth of Co nanostructures on a strained $\mathrm{Au}(111)$ surface
}

Paolo Campiglio, Vincent Repain, Cyril Chacon, Olivier Fruchart, Jérôme

Lagoute, Yann Girard, Sylvie Rousset

\section{- To cite this version:}

Paolo Campiglio, Vincent Repain, Cyril Chacon, Olivier Fruchart, Jérôme Lagoute, et al.. Quasi unidimensional growth of Co nanostructures on a strained $\mathrm{Au}(111)$ surface. Surface Science: A Journal Devoted to the Physics and Chemistry of Interfaces, 2011, 605, pp.1165. 10.1016/j.susc.2011.03.019 . hal-00548607

\section{HAL Id: hal-00548607 https://hal.science/hal-00548607}

Submitted on 20 Dec 2010

HAL is a multi-disciplinary open access archive for the deposit and dissemination of scientific research documents, whether they are published or not. The documents may come from teaching and research institutions in France or abroad, or from public or private research centers.
L'archive ouverte pluridisciplinaire HAL, est destinée au dépôt et à la diffusion de documents scientifiques de niveau recherche, publiés ou non, émanant des établissements d'enseignement et de recherche français ou étrangers, des laboratoires publics ou privés. 


\title{
Quasi unidimensional growth of Co nanostructures on a strained $\mathrm{Au}(111)$ surface
}

\author{
P. Campiglio ${ }^{\mathrm{a}, \mathrm{b}}$, V. Repain ${ }^{\mathrm{a}}$, C. Chacon ${ }^{\mathrm{a}}$, O. Fruchart ${ }^{\mathrm{c}}$, J. Lagoute $^{\mathrm{a}}$, Y. \\ Girard $^{\mathrm{a}}$, S. Rousset ${ }^{\mathrm{a}}$ \\ ${ }^{a}$ Laboratoire Matériaux et Phénomènes Quantiques, Université Paris Diderot $\mathcal{E}$ CNRS, \\ UMR 7162, case courrier 7021, F-75205 Paris Cedex 13, France \\ ${ }^{b}$ Department of Materials Science, Università degli Studi di Milano-Bicocca, Via R. \\ Cozzi 53 Milano, 20125, Italy \\ ${ }^{c}$ Institut NÉEL, CNRS \& Université Joseph Fourier, F-38042 Grenoble Cedex 9, France
}

\begin{abstract}
We investigated to which extent a uniaxially-strained $\mathrm{Au}(111)$ surface, thereby displaying linear reconstructions, may be used as a template for the growth of elongated nanostructures. Cobalt has been used as a deposition material, and two routes have been followed satisfactorily. The first one consists in the control of diffusion through the temperature of deposition. The second one consists in a first step of platinum seeding in the reconstruction, followed by cobalt deposition with nucleation on the platinum seeds.

Keywords:

Self-assembly, Surface diffusion, Low index single crystal surfaces, Metal-metal magnetic thin film structures, Scanning tunneling microscopy, Cobalt
\end{abstract}

\section{Introduction}

The ability to organize matter into objects with regular size, shape and spacing down to the atomic scale represents a cornerstone for nanoscience and nanotechnology. Top-down methods, mainly based on lithographic techniques, are time-consuming, expensive and in most of the cases limited to sizes above a few tens of nanometers. In this context a promising alternative, which has attracted an increasing attention, relies on bottom-up approaches.

Email address: vincent.repain@univ-paris-diderot.fr (V. Repain) 
These exploit diffusion and aggregation processes specific to each system, to drive the assembly of atoms and molecules in nanostructures [1]. Such routes are particularly interesting when using pre-existing self-organized template surfaces [2], which guide the growth and yield ordered arrays of nanodots. One major output of using surface templates is that the resulting nanostructures display a narrow size distribution. The guiding effect of the template may often be applied to several materials, which otherwise would not give rise to an organized array. For metal surfaces, a model template is provided by the $\mathrm{Au}(111)$ surface. This displays a so-called herringbone reconstruction [3] at the elbows of which many materials may nucleate and form ordered arrays of dots [4]. The distance between nearest-neighbor dots is about 7 $\mathrm{nm}$, and the metallic clusters display a nearly mono-disperse lateral size up to a few nanometers in diameter. On the one hand this peculiarity has allowed the study of fundamental physical properties (notably the magnetic properties such as magnetic anisotropy) of low dimensional model systems $[5,6,7]$. On the other hand, the $\mathrm{Au}(111)$ surface lacks of versatility since its surface pattern makes difficult to control the dots shape. In particular, the natural surface of $\mathrm{Au}(111)$ does not allow the versatile growth of nanowires, because its template is two-dimensional with a rectangular unit cell. In fact local nanowires can be achieved for certain materials in a narrow range of coverage when percolation sets in [8] but they are not macroscopically oriented along the same direction. So far, oriented nanowires have been grown on anisotropic substrates like (110) surface of fcc metals $[9,10]$, vicinal surfaces [11], molecular networks [12] or ripples obtained by etching under kinetic limitations $[13,14]$. The use of dense surface for the growth of nanowires would be a technological improvement since they are the most common surfaces observed during thin films growth. $\mathrm{Au}(111)$ appears to be a good candidate for anisotropic dense surface as it had been shown that when subjected to a uniaxial strain a $\mathrm{Au}(111)$ thin film may display a purely linear reconstruction, instead of the usual herringbone [15]. While initially performed in situ on a bent mica sheet, providing only a temporary control of the strain, recently linear reconstructions on $\mathrm{Au}(111)$ could be stabilized by uniaxial epitaxial strain on a body-centered cubic W(110) underlayer film $[16]$.

In this paper, we investigate to what extent this new reconstruction may be used as a template for self-organizing wires or chains of dots. We successfully produced elongated-shaped nanodots of cobalt following two routes. The first one relies on the temperature control over the diffusion of Co adatoms while 
the second one exploits the gold-platinum surface alloy to create a prepatterned surface with controlled nucleation sites.

\section{Experimental details}

The samples consist of a $\mathrm{Au}(111)[8 \mathrm{~nm}] / \mathrm{W}(110)[10 \mathrm{~nm}]$ thin film epitaxially grown on Sapphire (1120) by pulsed-laser deposition in a first ultra-high vacuum chamber. The base pressure is $3 \times 10^{-11} \mathrm{mbar}$ and the typical deposition rate is $1 \AA / \mathrm{s}[17]$. The samples were transferred through air to another chamber for the STM studies. The base pressure is better than $10^{-10}$ mbar. Here the sample is cleaned with repeated cycles of grazingincidence bombardment with argon ions (typically for $30 \mathrm{~s}$ with an incidence angle $\alpha=45^{\circ}$ at an Ar pressure of $2 \times 10^{-6}$ mbar and a voltage of $1 \mathrm{kV}$ ) followed by annealing at $650{ }^{\circ} \mathrm{C}$. Cobalt and platinum were evaporated by electron bombardment heating of high purity rods (99.99\%). Typical flux rates are $0.2 \mathrm{ML}$ (monolayer)/min and $0.02 \mathrm{ML} / \mathrm{min}$ for $\mathrm{Co}$ and $\mathrm{Pt}$ respectively. The deposition is performed on the sample placed in a Omicron Nanotechnology (C)T-STM (operating range from $50 \mathrm{~K}$ to $500 \mathrm{~K}$ ). The cobalt and platinum coverage $\left(\theta_{\mathrm{Co}}\right.$ and $\left.\theta_{\mathrm{Pt}}\right)$ are determined on the basis of STM images, assuming a pseudomorphic growth with the $\mathrm{Au}(111)$ substrate. The pressure during deposition is always maintained below $1 \times 10^{-10} \mathrm{mbar}$. Morphological studies are made by in situ STM at the temperature of the deposition.

\section{Results and discussion}

\subsection{Au(111) with unidirectional reconstruction}

The driving force for $\mathrm{Au}(111)$ surface reconstruction is the surface tensile stress that would occur at the surface of $\mathrm{Au}(111)$, if it were unreconstructed. In response to this stress the surface layer spontaneously densifies along the $\langle 1 \overline{1} 0\rangle$ directions, adding one extra atom every 22 atoms of the underlying layer with bulk lattice parameters, giving rise to the $22 \times \sqrt{3}$ reconstruction $[18,19]$. Inside the unit cell the atoms are arranged in fcc and hcp-type stacking domains separated by two stacking fault lines (or discommensuration lines). At larger scale a so-called herringbone reconstruction is observed: the $22 \times \sqrt{3}$ domains bend of $120^{\circ}$ and form a mesoscopically ordered pattern with a typical period of $25 \mathrm{~nm}$ [3]. This secondary superstructure arises from more delicate long-range elastic interactions mediated by the substrate, to relax a compression otherwise unidirectional [20]. Because of such central 
role played in the reconstruction, it is clear that stress is a key parameter to handle if one wishes to guide the surface towards new arrangements.

To this purpose we used a multilayer sample (schematically shown in Fig. 1) in which a uniaxial in-plane strain is imposed through the epitaxial relation with an underlayer with a crystalline uniaxial symmetry [16].

In short, the substrate we choose for our sample is Sapphire (1120) surface, over which a very thin film $(0.7 \mathrm{~nm})$ of Mo and a thicker one $(10 \mathrm{~nm})$ of $\mathrm{W}$ are grown and annealed such as to yield a single-crystalline body-centered layer with an atomically-smooth surface, with terraces of width several hundreds of nanometers [17]. Both Mo and W are bcc metals that during the growth expose the (110) surface characterized by a centered rectangular bidimensional lattice. Due to its anisotropy this layer induces a uniaxial strain in the $\mathrm{Au}$ layer (deposited at room temperature and annealed at $400{ }^{\circ} \mathrm{C}$ ), lifting the original three-fold symmetry and inducing a preferential orientation of the compressed [1ํㅣ ] direction [16]. The initial thickness of the Au layer is $8 \mathrm{~nm}$.

In Fig. 2a an image of the uniaxial $\mathrm{Au}(111)$ surface is reported. The brighter lines are the discommensuration lines that run along the $[11 \overline{2}] \mathrm{di}$ rection, while in the close-packed [110] direction hcp and fcc atomic stacking regularly alternate. As can be clearly seen, the herringbone reconstruction is suppressed and the purely linear reconstruction is stable over the whole sample surface. The stacking faults lines are characterized by a corrugation height of $0.17 \pm 0.02 \AA$ while the distance between two adjacent maxima is close to $57 \AA$, in contrast from the value observed for the reconstruction of $\mathrm{Au}(111)$ single-crystal surface. Indeed, the supercell that can be identified

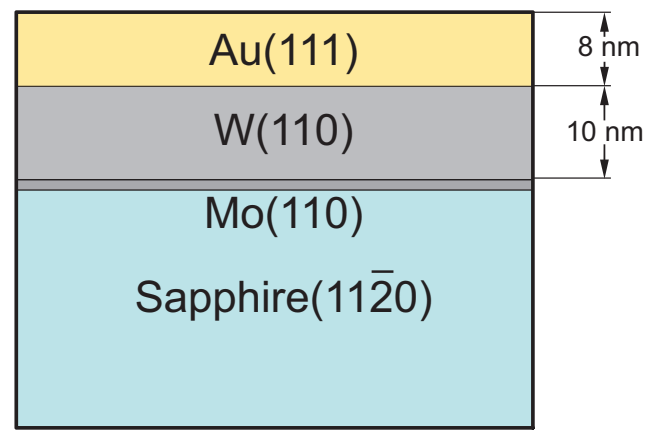

Figure 1: Schematic structure of the multilayer sample employed. The thickness of the Mo layer is $0.7 \mathrm{~nm}$. 

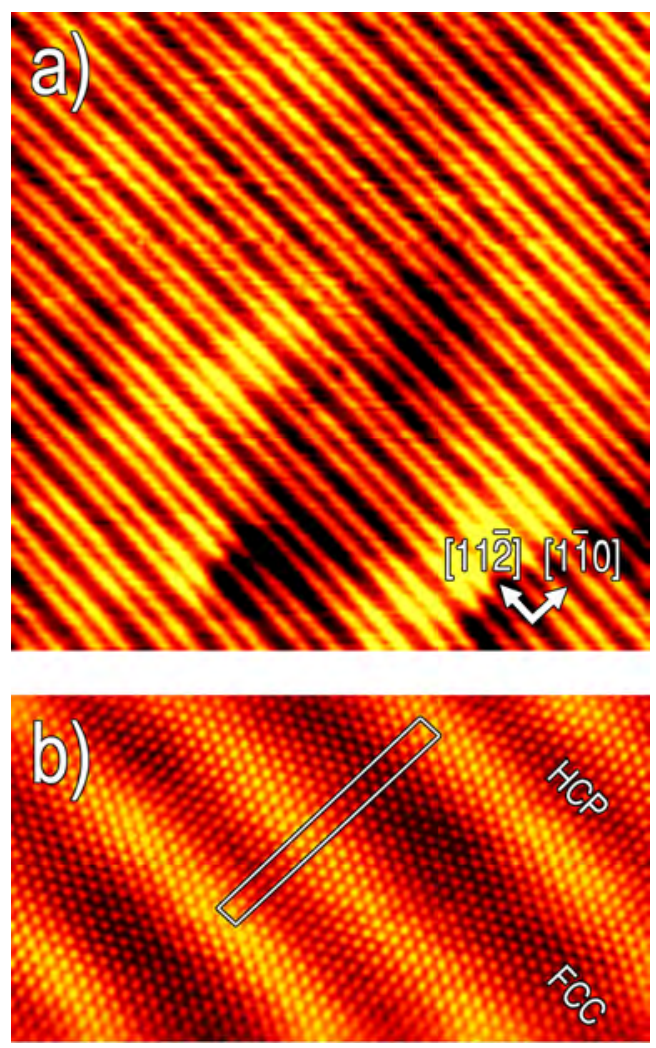

Figure 2: (a) Room temperature STM image showing the unidirectional reconstruction of the stressed $\mathrm{Au}(111)$ surface $\left(85 \times 85 \mathrm{~nm}^{2}\right)$. (b) Drift compensated atomic resolution STM image, with the unit cell highlighted $\left(15 \times 8 \mathrm{~nm}^{2}\right)$.

in Fig. 2b shows that the periodicity is drifted to 20 atomic positions.

At larger scale (Fig. 3a) some defects of the reconstruction also appear, mainly due to surface morphology. The most visible signatures in STM images comes from screw dislocations that appear as emerging steps. Edge dislocations leave weaker footprints on the morphology, but are responsible for important deviations from the purely linear reconstruction. At the surface, edge dislocations split into two partial dislocations that elastically repel each other and form a stacking fault ribbon that appear like a short step segment high one third of the full step height. These defects have been seldomly seen on single crystal $\mathrm{Au}(111)$ surface [21]. More particularly, on the present sample these stacking fault ribbons are commonly arranged in sequences, like shown in Fig. 3b, probably arising from grain boundaries. 

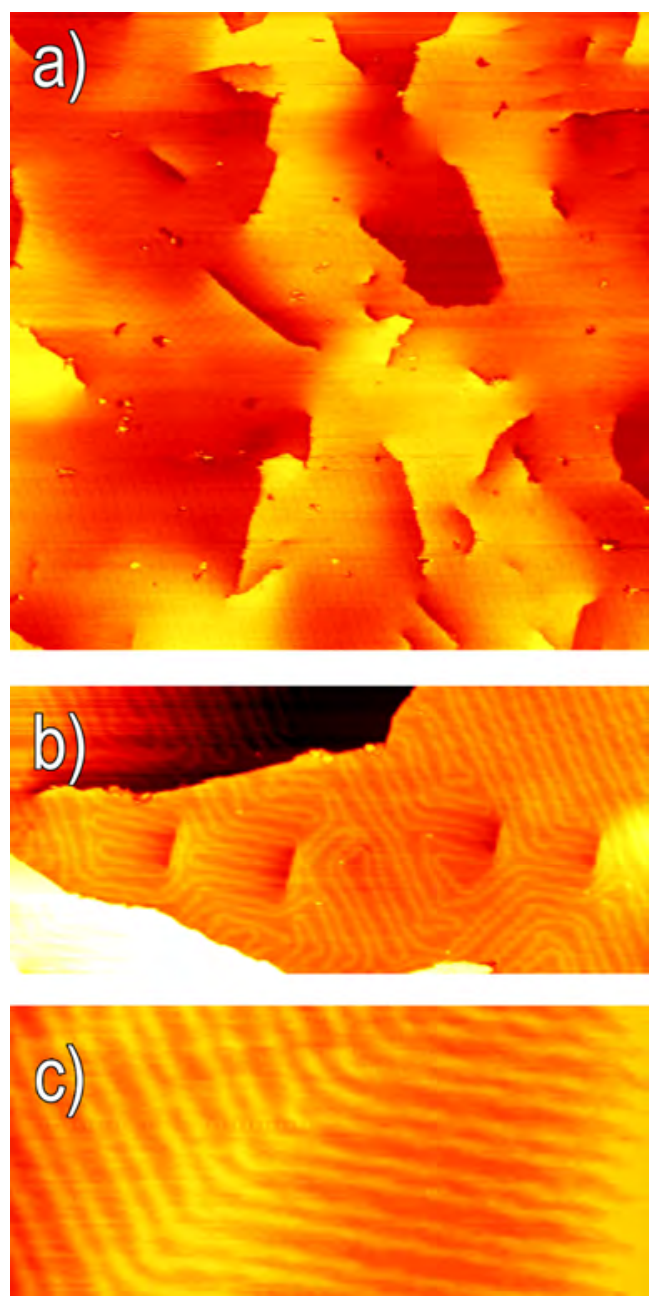

Figure 3: STM images of the $\mathrm{Au}(111)$ thin film surface (a) Large scale showing the presence of different defects $\left(360 \times 360 \mathrm{~nm}^{2}\right)$. (b) Effect of a sequence of stacking fault ribbons on $\mathrm{Au}(111)$ reconstruction $\left(130 \times 60 \mathrm{~nm}^{2}\right)$. (c) The reconstruction deviates from its unidimensional character and turns of $120^{\circ}$ as in the herringbone reconstruction $(60 \times$ $30 \mathrm{~nm}^{2}$ ). 
When the reconstruction deviates from its unidimensional character, it turns of $120^{\circ}$ as in the herringbone reconstruction (Fig. 3c). Naturally, the reconstruction elbows constitute the main trap for adatoms and represent the principal difficulty to overcome in order to obtain a homogeneous growth.

\subsection{Growth of Co on $A u(111)$}

Fig. 4 shows STM images of the $\mathrm{Au}(111)$ surface with unidirectional reconstruction onto which Co was deposited at low coverage $\left(\theta_{\mathrm{Co}}\right)$ in a wide range of temperatures $(50 \mathrm{~K}<T<300 \mathrm{~K})$. When the deposition of $0.25 \mathrm{ML}$ of Co is carried out at room temperature (Fig. 4a) the diffusion channels are clearly activated along all directions and thus the diffusion of adatoms is essentially isotropic. Due to the long diffusion length at this temperature, the adatoms are eventually pinched at defects of the surface. These are mainly dislocations or random atomic impurities; their role must be underlined in the present case because of the thinness of the Au layer that requires reduced time of Ar sputtering as compared to a single crystal. As a consequence, the Co growth proceeds very differently compared to the growth on herringbone reconstruction [22]. While the profile analysis indicates that the dots retain the usual bilayer height, the absence of periodic elbows in the reconstruction makes the dots loose both their regular spacing and their narrow size distribution.

To obtain an anisotropic diffusion we have reduced the temperature of the substrate at $105 \mathrm{~K}$ for the deposition. the temperature of deposition is not very critical, as the processes described below were found to be identical at $135 \mathrm{~K}$. As can be seen in Fig. 4b, Co $\left(\theta_{\mathrm{Co}}=0.15 \mathrm{ML}\right)$ forms dots elongated along the reconstruction direction, long up to $20 \mathrm{~nm}$ and with a mean aspect ratio equal to 5 . The nucleation of this kind of dots takes place on the fcc zone of the reconstruction. From these observations we can deduce that at this temperature the diffusion is anisotropic, with the path of adatoms along a direction parallel to the discommensuration lines being favored. The discommensuration lines seem to act as energy barrier, repelling the diffusion of Co adatoms as observed for $\mathrm{Al}$ [23]. This contrasts with molecular dynamics simulations, which predicted a more favorable diffusion perpendicularly to the discommensuration lines, followed by slower diffusion on the top and along the discommensurations [24]. They act as attractors and constitute a favored path to kink sites, where Co nucleates and grows [25]. However, the sites along the sides of the discommensuration are found to show higher adsorption energies then fcc and hcp sites and thus could explain their repul- 

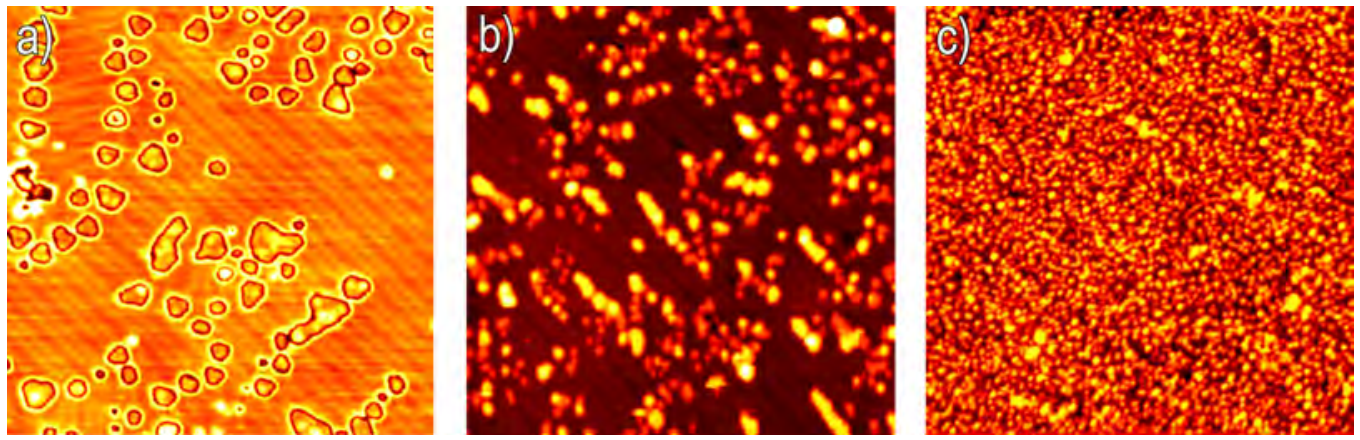

Figure 4: STM images of Co deposited at different temperatures on $\mathrm{Au}(111)$ with unidirectional reconstruction $\left(90 \times 90 \mathrm{~nm}^{2}\right)$. All the images are recorded at the deposition temperature. (a) $T=296 \mathrm{~K}, \theta_{\mathrm{Co}}=0.25 \mathrm{ML}$, isotropic diffusion; this image has been treated in order to enhance the contrast on the substrate. (b) $T=105 \mathrm{~K}, \theta_{\mathrm{Co}}=0.15 \mathrm{ML}$, anisotropic diffusion that leads to elongated dots. (c) $T=50 \mathrm{~K}, \theta_{\mathrm{Co}}=0.15 \mathrm{ML}$, postnucleation regime.

sive character with respect to diffusing Co adatoms [26]. Due to the reduced mobility of adatoms, impurities and reconstruction defects constitute even more efficient traps than at room temperature. As a result, besides elongated dots also very small clusters of Co nucleate, leading to a wide distribution of dot size and shape. It is the same type of defects, including impurities, that are the source of nucleation at $300 \mathrm{~K}$ (Fig. 4). We believe that impurities are not only intrinsic, but are also due to the fact that the same template has been used several times to perform experiments at various temperatures. The etching procedure applied between each deposition to sputter away the Co atoms, was minimized so that the entire Au film (total initial thickness $8 \mathrm{~nm}$ ) is not fully etched away. Probably this leaves Co impurities at the surface, which upon annealing are known to embed themselves in the uppermost plane of $\mathrm{Au}$ atoms, creating nucleations sites for the next deposition [27]. It is therefore likely that deposition at $105 \mathrm{~K}$ on a fresh template would yield a more homogeneous growth of elongated dots.

As expected, if we further decrease the temperature of deposition down to $50 \mathrm{~K}\left(\theta_{\mathrm{Co}}=0.15 \mathrm{ML}\right)$, the diffusion of adatoms is essentially completely frozen and a post nucleation regime is observed, giving rise to frozen disorder. As shown in Fig. 4c, monolayer-thick dots with lateral size of few Angströms nucleate at spatially random locations with a high density, in a rather similar way than on $\mathrm{Au}(111)$ surface with herringbone reconstruction [28].

The temperature control over the diffusion barrier is an elegant way to drive 
the self-assembly of nanostructures, and we successfully employed it to grow elongated dots also in the present case. However the high density of intrinsic and extrinsic defects on the surface disturbs the diffusion of Co adatoms, preventing a homogeneous growth. In order to have a better control on the number of nucleation centers on the surface, Pt seeding appears to be a suitable candidate to elaborate a prepattening of the surface [29]. Pt is miscible with $\mathrm{Au}$ and previous studies showed that when deposited at room temperature on $\mathrm{Au}(111)$, Pt substitute $\mathrm{Au}$ atoms in the surface layer [30]. Since at room temperature the $\mathrm{Au}(111)$ surface could host a maximum of $0.03 \mathrm{ML}$ of $\mathrm{Pt}$, we increased the substrate temperature to $400 \mathrm{~K}$ to favor the exchange of $\mathrm{Pt}$ with $\mathrm{Au}$ atoms. Fig. 5a shows a room temperature STM image of $0.05 \mathrm{ML}$ of $\mathrm{Pt}$ deposited on $\mathrm{Au}(111)$ at $400 \mathrm{~K}$. The embedded Pt atoms are seen darker than the surrounding Au atoms, with an apparent height different of $0.2 \AA$ with respect to the mean surface level. As a detrimental consequence of the alloying, Au atoms are ejected from the surface and forms dots of $2.35 \AA$ high that may perturb the regularity of the reconstruction. More importantly, Pt atoms alloying does not occur randomly on the surface but takes place preferentially in the discommensuration regions (See inset of Fig. 5a). Thus the surface alloy could be regarded as a patterned surface in which $\mathrm{Pt}$ atoms constitute trapping sites that may permit to trigger the nucleation of Co. The ability of a seed layer to guide the growth of materials that otherwise do not show any order has been shown also for $\mathrm{Pd}$ on $\mathrm{Ni}_{3} \mathrm{Al}(111)$ [31] or Ir on graphene/Ir(111) [32] This is confirmed in Fig. $5 \mathrm{~b}$, where $0.15 \mathrm{ML}$ of Co where deposited at room temperature on $\mathrm{Au}(111)$ seeded with Pt. Since the diffusion of adatoms is quenched by the presence of $\mathrm{Pt}$ atoms, Co dots nucleate with a high density in the form of monolayer dots with a mean lateral dimension of $1 \mathrm{~nm}$. In the case of such small dots it has been shown that it is energetically more favorable to adopt a monolayer rather than a bilayer height in order to maximize the atomic coordination [33]. The Co follows the order imposed by the previous Pt deposition and forms hemispherical dots aligned along the reconstruction lines and not on the fcc stacking regions as we observed in the low-temperature growth. In particular Co nucleates alternatively in one over two discommensuration regions and thus the distance between neighbor nanodots is $7 \mathrm{~nm}$. This is due to the fact that preferentially the alloying of $\mathrm{Pt}$ with $\mathrm{Au}$ takes place alternatively in one single discommensuration lines of the reconstruction, as can be seen also in Fig. 5a. When $\theta_{\text {Co }}$ is increased, dots grow first vertically, adopting the usual bilayer height, and then laterally (Fig. 5c). During this 

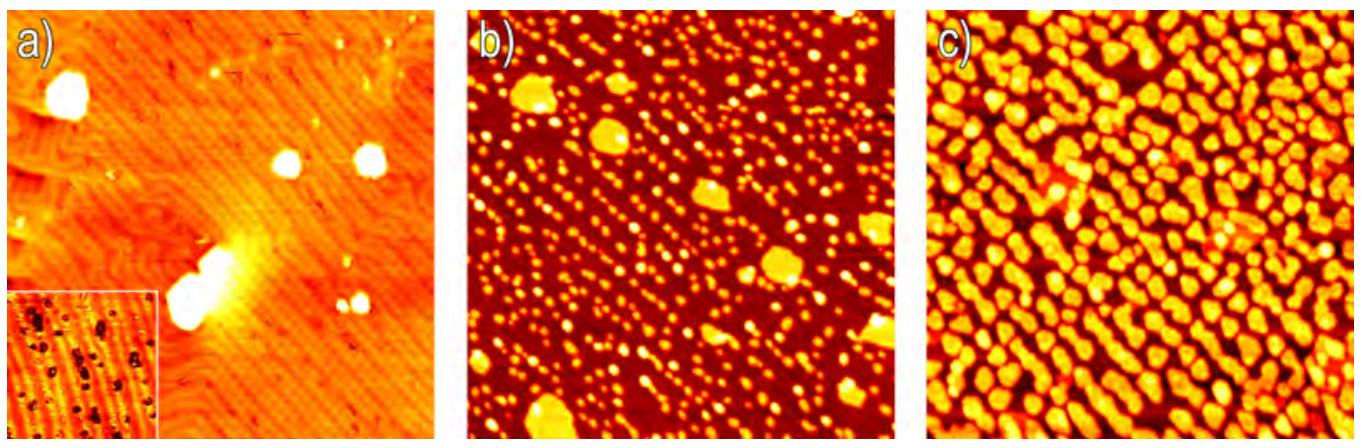

Figure 5: (a) STM image of $\mathrm{Pt}\left(\theta_{\mathrm{Pt}}=0.05 \mathrm{ML}\right)$ deposited at $400 \mathrm{~K}$ on $\mathrm{Au}(111)$ with unidirectional reconstruction; the $\mathrm{Pt}$ is embedded in the surface and follows the reconstruction lines. In inset, zoom $\left(32 \times 32 \mathrm{~nm}^{2}\right)$ showing dark patches corresponding to Pt inserted atoms. (b) $\left(\theta_{\mathrm{Co}}=0.15 \mathrm{ML}\right)$ and $(\mathrm{c})\left(\theta_{\mathrm{Co}}=1.1 \mathrm{ML}\right)$, STM images of Co deposited on $\mathrm{Pt} / \mathrm{Au}(111)$ : $\mathrm{Pt}$ acts like preferential nucleation sites for Co that forms small and separated dots at low Co coverage (b), that coalesce when the Co coverage is increased (c). All the images are $100 \times 100 \mathrm{~nm}^{2}$ and recorded at room temperature.

process the clusters percolate and, thanks to the small distance between dots on the same reconstruction line, form dots often very elongated along the reconstruction direction, with an aspect ratio up to 8 . In some other cases even if two dots come very close together, the percolation does not take place probably due to an incommensurate growth of the dots [34]. This is observed even more systematically on $\mathrm{Au}(111)$ with herringbone reconstruction, and it is why the magnetic transition of Co nanodots to ferromagnetic films happen only at $1.5 \mathrm{ML}$. The problem of incommensurate growth in this case seems to be less stringent and several dots can coalesce together, as can be seen in Fig. 5c. Another relevant difference with $\mathrm{Au}(111)$ with herringbone is that the coalesce takes place in the same direction also at a macroscopic scale. This is due to the fact that the direction of the reconstruction is maintained fixed all over the sample, if surface defects are neglected. As an important consequence, the nanodots are preferentially elongated along the same axis. Significantly, this should permit to macroscopically measure the properties of such anisotropic objects along different directions. When applied to freshly re-prepared samples of uniaxial $\mathrm{Au}(111)$ reconstruction, the two procedures applied above (low temperature deposition or Pt seeding) are expected to provide a much cleaner template, efficient to fabricate uniaxial nanostructures of various materials on a macroscopic scale. It is important to stress that no self-organized linear template with such a large period is currently 
available starting from nominally-flat surfaces, i.e. compatible with a standard wafer technology. This may open the way to the use of such templates for the investigation of physical properties of nanowires in a yet unexplored range of width.

\section{Conclusions}

In this work we employ a uniaxially strained $\mathrm{Au}(111)$ surface, whose reconstruction is lifted to purely linear thanks to the epitaxial constraints of an underlying multilayer sample. We investigated the capability of this surface to act as a template to obtain unidimensional nanostructures. To achieve this, we focalized our attention on the growth of cobalt in submonolayer coverages. Elongated dots with an aspect ratio up to 8 were successfully obtained by following two different routes. In the first one we use the temperature to control the diffusion of adatoms across the surface. Below about $150 \mathrm{~K}$ the diffusion channel across the reconstruction is blocked and the anisotropic diffusion results in an elongated growth. Alternatively, we have successfully imposed order nucleation sites. This was done with the predeposition of a seed layer of Pt that forms a surface alloy with Au preferentially on the discommensuration lines. We believe that both these routes may be used to realize elongated nanodots of various materials ( $\mathrm{Mn}, \mathrm{Fe}, \mathrm{Ni}, \mathrm{Pd}, \mathrm{Pt} . .$.$) . The$ ability to control the diffusion and aggregation of adatoms combined with the skill to tailor a given surface structure is a powerful way to control the growth of metal nanostructures. This objective is of high importance in order to understand and exploit their physical properties.

\section{Acknowledgement}

The authors thank A. Sassella for fruitful discussions. V.R. acknowledges financial support from contract ANR-ETNAA (ANR-NANO-018-04)

and Insitut Universitaire de France. O.F. acknowledges financial support from contract ANR-05-NANO-073.

\section{References}

[1] J. V. Barth, G. Costantini, K. Kern, Engineering atomic and molecular nanostructures at surfaces, Nature 437 (2005) 671. 
[2] S. Rousset, B. Croset, Y. Girard, G. Prévot, V. Repain, S. Rohart, Selforganized epitaxial growth on spontaneously nano-patterned templates, C. R. Physique 6 (2005) 33.

[3] J. V. Barth, H. Brune, G. Ertl, R. J. Behm, Scanning tunneling microscopy observations on the reconstructed $\mathrm{Au}(111)$ surface: Atomic structure, long-range superstructure, rotational domains, and surface defects, Phys. Rev. B 42 (1990) 9307.

[4] D. D. Chambliss, R. J. Wilson, S. Chiang, Nucleation of ordered Ni island arrays on $\mathrm{Au}(111)$ by surface-lattice dislocations, Phys. Rev. Lett. 66 (1991) 1721.

[5] H. A. Dürr, S. S. Dhesi, E. Dudzik, D. Knabben, G. V. Laan, J. Goedkoop, F. U. Hillebrecht, Spin and orbital magnetization in self-assembled Co clusters on $\mathrm{Au}(111)$, Phys. Rev. B 59 (1999) R701.

[6] Y. Nahas, V. Repain, C. Chacon, Y. Girard, J. Lagoute, G. Rodary, J. Klein, S. Rousset, H. Bulou, C. Goyhenex, Dominant role of the epitaxial strain in the magnetism of core-shell $\mathrm{Co} / \mathrm{Au}$ self-organized nanodots, Phys. Rev. Lett. 103 (6) (2009) 067202.

[7] S. Rohart, P. Campiglio, V. Repin, Y. Nahas, C. Chacon, Y. Girard, J. Lagoute, A. Thiaville, S. Rousset, Spin-wave-assisted thermal reversal of epitaxial perpendicular magnetic nanodots, Phys. Rev. Lett. 104 (13) (2010) 137202.

[8] P. Ohresser, N. Brookes, S. Padovani, F. Scheurer, H. Bulou, Magnetism of small Fe clusters on $\mathrm{Au}(111)$ studied by x-ray magnetic circular dichroism, Phys. Rev. B 64 (2001) 104429.

[9] H. Röder, E. Hahn, H. Brune, J.-P. Bucher, K. Kern, Building one- and two-dimensional nanostructures by diffusion-controlled aggregation at surfaces, Nature 366 (1993) 141.

[10] H. Sahaf, L. Masson, C. Léandri, B. Aufray, G. L. Lay, F. Ronci, Formation of a one-dimensional grating at the molecular scale by self-assembly of straight silicon nanowires, Appl. Phys. Lett. 90 (2007) 263110. 
[11] P. Gambardella, M. Blanc, H. Brune, K. Kuhnke, K. Kern, Onedimensional metal chains on Pt vicinal surfaces, Phys. Rev. B 61 (2000) 2254 .

[12] X.-D. Ma, D. I. Bazhanov, O. Fruchart, F. Yildiz, T. Yokoyama, M. Przybylski, V. S. Stepanyuk, W. Hergert, J. Kirschner, Strain relief guided growth of atomic nanowires in a $\mathrm{Cu}_{3} \mathrm{~N}-\mathrm{Cu}(110)$ molecular network, Phys. Rev. Lett. 102 (20) (2009) 205503.

[13] T. Aste, U. Valbusa, Ripples and ripples: from sandy deserts to ionsputtered surfaces, New J. Phys. 7 (2005) 122.

[14] B. Borca, O. Fruchart, P. David, A. Rousseau, C. Meyer, Kinetic selforganization of trenched templates for the fabrication of versatile ferromagnetic nanowires, Appl. Phys. Lett. 90 (2007) 142507.

[15] O. Schaff, A. Schmid, N. Bartelt, J. de la Figuera, R. Hwang, In-situ STM studies of strain-stabilized thin-film dislocation networks under applied stress, Mater. Sci. Eng. A 319 (2001) 914.

[16] O. Fruchart, H. Tolentino, M. D. Santis, C. Goyhenex, H. Bulou, C. Clavero, M. Przybylski, J. Kirschner, to be published.

[17] O. Fruchart, P. Jubert, M. Eleoui, F. Cheynis, B. Borca, P. David, V. Santonacci, A. Liénard, M. Hasegawa, C. Meyer, Growth modes of $\mathrm{Fe}(110)$ revisited: a contribution of self-assembly to magnetic materials, J. Phys.: Condens. Matter. 19 (2007) 053001.

[18] U. Harten, A. Lahee, J. Toennies, C. Wöll, Observation of a soliton reconstruction of au(111) by high-resolution helium-atom diffraction, Phys. Rev. Lett. 54 (1985) 2619.

[19] A. Sandy, S. Mochrie, D. Zehner, K. Huang, D. Gibbs, Structure and phases of the au(111) surface: X-ray scattering measurements, Phys. Rev. Lett. 43 (1991) 4667.

[20] S. Narasimhan, D. Vanderbilt, Elastic stress domains and the herringbone reconstruction on $\mathrm{Au}(111)$, Phys. Rev. Lett. 69 (1992) 1564.

[21] J. Engbæk, J. Schiøtz, Atomic structure of screw dislocations intersecting the $\mathrm{Au}(111)$ surface: A combined scanning tunneling microscopy and molecular dynamics study, Phys. Rev. B 74 (2006) 195434. 
[22] B. Voigtländer, G. Meyer, N. Amer, Epitaxial growth of thin magnetic cobalt films on $\mathrm{Au}$ (111) studied by scanning tunneling microscopy, Phys. Rev. B 44 (18) (1991) 10354.

[23] B. Fischer, H. Brune, J. Barth, A. Fricke, K. Kern, Nucleation kinetics on inhomogeneous substrates: $\mathrm{Al} / \mathrm{Au}$ (111), Phys. Rev. Lett. 82 (8) (1999) 1732.

[24] H. Bulou, Atomic diffusion on nanostructured surfaces, Superlatt. Microstr 44 (2008) 533.

[25] H. Bulou, C. Massobrio, Dynamical behavior of co adatoms on the herringbone reconstructed surface of au (111), Superlattice Microst. 36 (2004) 305.

[26] C. Goyhenex, H. Bulou, Theoretical insight in the energetics of Co adsorption on a reconstructed $\mathrm{Au}(111)$ substrate, Phys. Rev. B 63 (2001) 235404.

[27] S. Padovani, I. Chado, F. Scheurer, J. P. Bucher, Transition from zerodimensional superparamagnetism to two-dimensional ferromagnetism of Co clusters on Au(111), Phys. Rev. B 59 (1999) 71815.

[28] S. Padovani, F. Scheurer, J. Bucher, Burrowing self-organized cobalt clusters into a gold substrate, Europhys. Lett. 45 (1999) 327.

[29] Y. Nahas, V. Repain, C. Chacon, Y. Girard, S. Rousset, Interplay between ordered growth and intermixing of $\mathrm{Pt}$ on patterned Au surfaces, Surf. Sci. 604 (2010) 829.

[30] M. Pedersen, S. Helveg, A. Ruban, I. Stensgaard, E. Laegsgaard, J. K. Norskov, F. Besenbacher, How a gold substrate can increase the reactivity of a Pt overlayer, Surf. Sci. 426 (1999) 395.

[31] G. Hamm, C. Becker, C. Henry, Bimetallic Pd-Au nanocluster arrays grown on nanostructured alumina templates, Nanotechnology 17 (2006) 1943.

[32] A. N'Diaye, T. Gerber, C. Busse, J. Mysliveček, J. Coraux, T. Michely, A versatile fabrication method for cluster superlattices, New J. Phys. 11 (2009) 103045. 
[33] I. Chado, C. Goyhenex, H. Bulou, J. Bucher, Cluster critical size effect during growth on a heterogeneous surface, Phys. Rev. B 69 (2004) 085413.

[34] O. Fruchart, G. Renaud, A. Barbier, M. Noblet, O. Ulrich, J. Deville, F. Scheurer, J. Mane-Mane, V. Repain, G. Baudot, S. Rousset, X-ray super-cell crystallography of self-organized $\mathrm{Co} / \mathrm{Au}(111)$ deposits, Europhys. Lett. 63 (2003) 275. 